\title{
Management of Hypertension in Pregnancy as a Quality Indicator of Antenatal Care in Rural Tanzania
}

\author{
David P Urassa', ${ }^{1,3}$ Lennarth Nystrom², Anders Carlstedt', Gernard I Msamanga', Gunilla Lindmark ${ }^{3}$
}

\begin{abstract}
To assess the ability of antenatal care to manage hypertension in pregnancy, a cross-sectional study involving 379 pregnant women was conducted in 16 randomly selected antenatal clinics in Rufiji district of Tanzania. We observed necessary structural availability, provider client interaction, interviewed women attending antenatal clinics, and measured their blood pressure. Measurements made by observers and health workers were compared. One third of the women were not checked for hypertension and health workers detected only four out of twelve women with elevated blood pressure. There was disagreement in diagnosis of blood pressure as measured by health workers and observers. Only one woman with elevated blood pressure was managed appropriately. The low quality of screening and management of hypertension in pregnancy makes it less realistic to expect any impact of antenatal care programme in reducing morbidity or mortality due to hypertension in pregnancy. (Afr J Reprod Health 2003; 7[3]: 69- 76)
\end{abstract}

\section{RÉSUMÉ}

Traitement de l'hypertension pendant la grossesse comme un indice de qualité de soin prénatal en Tanzanie rurale. Pour évaluer la capacité de soin prénatal de traiter l'hypertension pendant la grossesse, nous avons mené une étude transversale qui a fait intervenir 379 femmes enceintes, dans 16 services de consultation prénatale selectionnés au hasard dans le district de Rufiji en Tanzanie. Nous avons remarqué la disponibilité structurale, l'interaction entre dispensateur et client, intérrogé les femmes qui allaient à la consultation prénatale et nous avons mésuré la pression artérielle. Les mesures faites par les observateurs et le personnel soignant ont été comparées. Un tiers des femmes n'ont pas été contrôlé pour l'hypertension et la personnel soignant n'ont dépisté que quatre femmes sur douze dont la pression artérielle était élévée. Il y avait une disparité entre le diagnostique de la pression artérielle faite par le personnel soignant et celui fait par les observateurs. Il n' y avait qu'une seule femme dont la pression artérielle était élevé que suivait un traitement approprié. Le bas niveau du dépistage et du traitement de l'hypertension dans la grossesse le rendent moins pratique de s'attendre à un impact quiconque de programme de service de consultation prénatale sur la reduction de la morbidité ou de la mortalité occasionnée par l'hypertension pendant la grossesse. (Rev Afr Santé Reprod 2003; 7[3]: 69-76)

KEY WORDS: Quality, antenatal care, programme evaluation, bypertension, pregnancy, Africa

${ }^{1}$ Department of Community Health, Mubimbili University College of Health Sciences (MUCHS), Dar es Salaam, Tanzania.

${ }^{2}$ Department of Public Health and Clinical Medicine Epidemiology, Umea University, Umea, Sweden. ${ }^{3}$ Department of Women's and Children's Health, International Maternal and Child Health (IMCH), University Hospital, Uppsala, Sweden. 


\section{Introduction}

Measurement of blood pressure is essential for diagnosis and management of hypertensive disorders in pregnant women. The major aim of regular blood pressure measurements during pregnancy is the early diagnosis of hypertensive complications, especially pre-eclampsia, since they are important causes of premature births, perinatal and maternal mortality. ${ }^{1}$ In the maternal and child health $(\mathrm{MCH})$ programme in Tanzania, hypertension in pregnancy is monitored during antenatal clinics according to the World Health Organization directives ${ }^{2}$ that have been translated into national guidelines. These guidelines are printed on the antenatal card issued to all women to record care in antenatal clinics. Blood pressure measurement is recommended for all pregnant women at all visits, and the referral guidelines require action to be taken by health workers when blood pressure is greater or equal to $140 / 90 \mathrm{mmHg}$. Appropriate treatment or referral depends on accurate measurements. ${ }^{3}$

Despite the high coverage (97\%) of antenatal care (ANC) attendance in Tanzania, ${ }^{4}$ maternal mortality is still reported to be high (572-960/ 100,000 live births). ${ }^{5-7}$ In Muhimbili National Hospital, the national referral hospital, hypertensive disorders are found in 16\% of women attending antenatal care. ${ }^{8}$ Hypertension/eclampsia was ranked as the third cause of maternal death in a communitybased study in Ilala district, Dar es Salaam in 1991$1993,{ }^{,}$contributing to $12 \%$ of deaths.

The co-existence of high antenatal coverage and high maternal mortality due to hypertension/ eclampsia casts doubts on the quality of screening and management of hypertension in pregnancy in this area. We aimed at investigating the extent to which routine antenatal care services succeeded in identifying and managing women at risk of preeclampsia or eclampsia using the antenatal card guidelines. Specifically, we assessed the percentage of antenatal clinics with adequate facilities for detecting hypertension in pregnancy, and the percentage of women examined for hypertension. We also recorded whether correct advice was given to hypertensive women and interviewed the clients on their experience with services provided.

\section{Subjects and Methods}

Study Site

A cross-sectional study was conducted in Rufiii, one of the Coast Region districts in Tanzania with an estimated population of 180,000 , based on the 1988 population census, and annual population growth of $2.3 \%$. The population is predominantly rural.

Antenatal care (ANC) is provided at two hospitals, four rural health centres (RHCs) and 48 dispensaries. The antenatal clinics are usually situated within the same building that provides curative care for non-pregnant women and adults. The study population was stratified by type of unit providing ANC. In two strata, hospitals and RHCs, all units were included in the sample, while in the third stratum 10 out of 48 dispensaries were randomly selected. This fulfils the recommendations to cover at least $25-30 \%$ of the health facilities in the area when assessing quality of care..$^{10,11}$

\section{Data Collection}

The research team consisting of the main investigator (DPU) and two qualified nurse midwives (research assistants) visited each facility for two days. An introductory meeting was held on the first day to familiarise with the clinic staff and to explain the aim of the study. The research team was introduced as not coming from the Ministry of Health and it was emphasised that the findings of the study would by no means be used against any staff member. On the second day, women attending ANC were observed and interviewed after informed verbal consent had been obtained from them. The first 30 women at the hospitals and RHCs and the first 20 women at dispensaries were invited to participate in the study. We got only 19 women in one dispensary on that day, thus a total of 379 women were investigated.

The first research assistant (an observer) was stationed in the consultation room and the second assistant by the exit door. Interaction between the health worker and the pregnant woman was observed after which blood pressure examination was performed by the observer. The second assistant interviewed mothers to find out what information 
and advice they received. She then assessed their satisfaction with the services.

The study used explicit measurements of three components of quality of care including structural process and outcome indicators relevant for detection and management of hypertension in pregnancy at ANC. An observation checklist was used to assess the availability of necessary structural facilities for detection of hypertension in pregnancy.

Using an observation check list, the observer took notes on the qualification of staff, whether a blood pressure measurement was performed, whether a feedback to the mother was given on risk factors detected and type of advice given or action taken. Women were examined twice for blood pressure, first by a health worker and then by an observer without having knowledge of results of the previous blood pressure measurement. Using a newly purchased aneroid sphygmomanometer the observer measured blood pressure on the right arm with the mother seated and her arm resting on a table at the same level as the heart, after a minimum of five minutes rest. The systolic and diastolic pressures were determined at Korotkoff phases I and $\mathrm{V}$ respectively.

For ethical reasons mothers found by the observer to have elevated blood pressure $(\geq 140$ / $90 \mathrm{mmHg}$ ) were sent back to the health worker with their blood pressure results for further investigations, treatment or referral after exit interviews. No attempt was made to follow the kind of action the women took after that.

To assess women's experience with the service, the second research assistant at exit interviewed mothers on the type of investigations or examinations done. She also asked if they had received any feedback on detected problems, any individual counselling or medication, and whether they were satisfied with the service received. Blood pressure readings from antenatal cards as reported by the health workers were also recorded.

\section{Standards}

The trained staff in the antenatal clinic had been exposed to midwifery during their basic training. In this study, an $\mathrm{MCH}$ aide with two years training in midwifery skills was considered to be the lowest cadre of trained staff. A pregnant woman was expected to attend a facility with a qualified staff and equipment for detection and management of hypertension in pregnancy as a structural requirement. Following the guideline in the antenatal card, women were expected to be examined for blood pressure, investigated for urinalysis and given the results of any investigation or examination even if the results were normal. It is recommended that all pregnant women with elevated blood pressure $\geq 140 / 90 \mathrm{mmHg}$ should be referred to the hospital level for expert care.

\section{Statistical Analysis}

The data were cleaned, coded, entered, checked for consistency and analysed using Epi Info. The agreement between health workers and observers was assessed by Kappa coefficient with value $(\mathrm{k})$ indicating $<0.20$ for poor, $0.21-0.40$ for fair, 0.410.60 for moderate, $0.61-0.80$ for substantial and $\geq$ 0.81 for almost perfect agreement between assessors. ${ }^{12}$ The operational cut-off level for blood pressure $>140 / 90 \mathrm{mmHg}$ was used for hypertension in pregnancy, suggesting the need for further investigation, treatment or referral according to the guidelines in the antenatal card. To test whether differences in prevalence between the health institutions were due to random variation or not a student t-test was used.

\section{Results}

We investigated $30 \%(16 / 54)$ of the health institutions that provide antenatal care at the dispensary, health centre and hospital levels in the district. Nine out of ten dispensaries had adequate number of qualified staff as well as a sphygmomanometer and a stethoscope (Table 1). Only one dispensary was capable of investigating albumin in urine and none had anti-hypertensive drugs. One of the four RHCs did not have either a stethoscope or a sphygmomanometer. Only the hospitals had all necessary infrastructure available for managing hypertension in pregnancy. Women who attended care services in institutions without qualified staff ( $\mathrm{n}=15$ ) or equipment to measure blood pressure $(\mathrm{n}=38)$ were obviously not screened. 
Table 1 Number of Health Institutions with Adequate Structural Facilities for Screening and Managing Hypertension in Antenatal Clinics

\begin{tabular}{lcccc}
\hline $\begin{array}{l}\text { Presence of specific structural } \\
\text { item }\end{array}$ & Health facility & & \\
& Dispensary & RHC & Hospital & Total \\
\hline Qualified staff & 9 & 4 & 2 & 15 \\
Sphygmomanometer & 9 & 3 & 2 & 14 \\
Stethoscope & 9 & 3 & 2 & 14 \\
Albumin investigation & 1 & 4 & 2 & 7 \\
Anti-hypertensives & 0 & 0 & 2 & 2 \\
Diazepam & 4 & 2 & 2 & 8 \\
Total No. of facilities & 10 & 4 & 2 & 16 \\
\hline
\end{tabular}

Table 2 Number and Percentage of Pregnant Women Screened by Observers (OB) and Health Workers (HW) who had Elevated Blood Pressure (EBP) and received Feedback

\begin{tabular}{|c|c|c|c|c|}
\hline & \multicolumn{3}{|c|}{ Health facility } & \multirow[t]{2}{*}{ Total $(\%$} \\
\hline & Dispensary & RHC & Hospital & \\
\hline Screened by OB & $189(100)$ & $129(100)$ & $61(100)$ & $379(100)$ \\
\hline Screened by HW & $104 \quad(55)$ & $99 \quad(77)$ & $45 \quad(74)$ & $248(65)$ \\
\hline Screened and informed by HW & $19(10.6)$ & $14(10.0)$ & $1(1.6)$ & $34(9.0)$ \\
\hline EBP by OB & $9 \quad(4.8)$ & $3(2.3)$ & $0 \quad(0)$ & $12(3.2)$ \\
\hline EBP by HW & $2(1.1)$ & $1(0.8)$ & $1(1.6)$ & $4(1.1)$ \\
\hline EBP and received counselling & $0 \quad(0)$ & $1(0.8)$ & $0 \quad(0)$ & $1(0.2)$ \\
\hline No. of women & 189 & 129 & 61 & 379 \\
\hline
\end{tabular}

Of the 379 pregnant women screened by the research team, two thirds $(65 \%)$ were screened for hypertension by health workers (Table 2). Among those institutions with necessary equipment and staff, the proportions of women screened by health workers were higher in hospitals (74\%) and RHCs $(77 \%)$ than dispensaries $(55 \%)(\mathrm{p}=0.02$ and $\mathrm{p}=$ 0.0001 respectively). Overall, $3.2 \%$ of women were found to have an elevated blood pressure as assessed by the observer, compared to $1.1 \%$ by health workers in the clinics.

The percentage of women detected with elevated blood pressure and those informed of their results are presented in (Table 2). About two thirds of women (248/379) were screened by both health workers and the observer. Among those screened by health workers, $9 \%$ were informed about their blood pressure results. Overall, the health workers detected only four out of 12 women in the total sample found by the observer to have elevated blood pressure, and only one woman received appropriate counselling. Health workers in dispensaries (10.6\%) and RHCs $(10.0 \%)$ were somehow more likely to give information on blood pressure results than those in hospitals $(1.6 \%)(\mathrm{p}=0.021)$.

The distribution of systolic and diastolic blood pressures as measured by health workers and an observer is shown in Figures 1a and 1b. Systolic 
blood pressure of $100 \mathrm{mmHg}$ and diastolic blood pressure of $60 \mathrm{mmHg}$ were the mode blood pressure value for women examined by health workers. The same women had mode systolic blood pressure of
120 and diastolic blood pressure of 80 when examined by the observer. Health workers generally recorded lower blood pressure values than the observer.

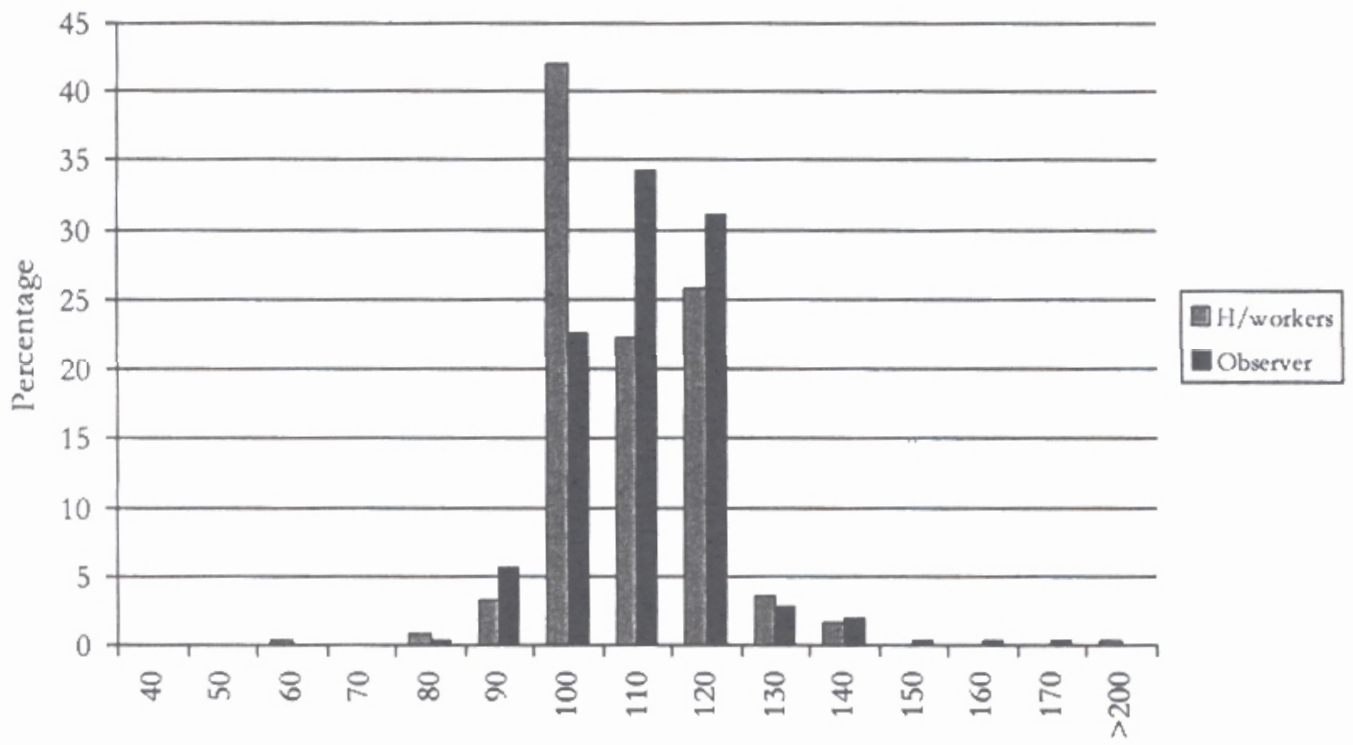

Systolic blood pressure

Figure 1a Distribution of Systolic Blood Pressure as recorded by Health Workers and Observer in 248 Women

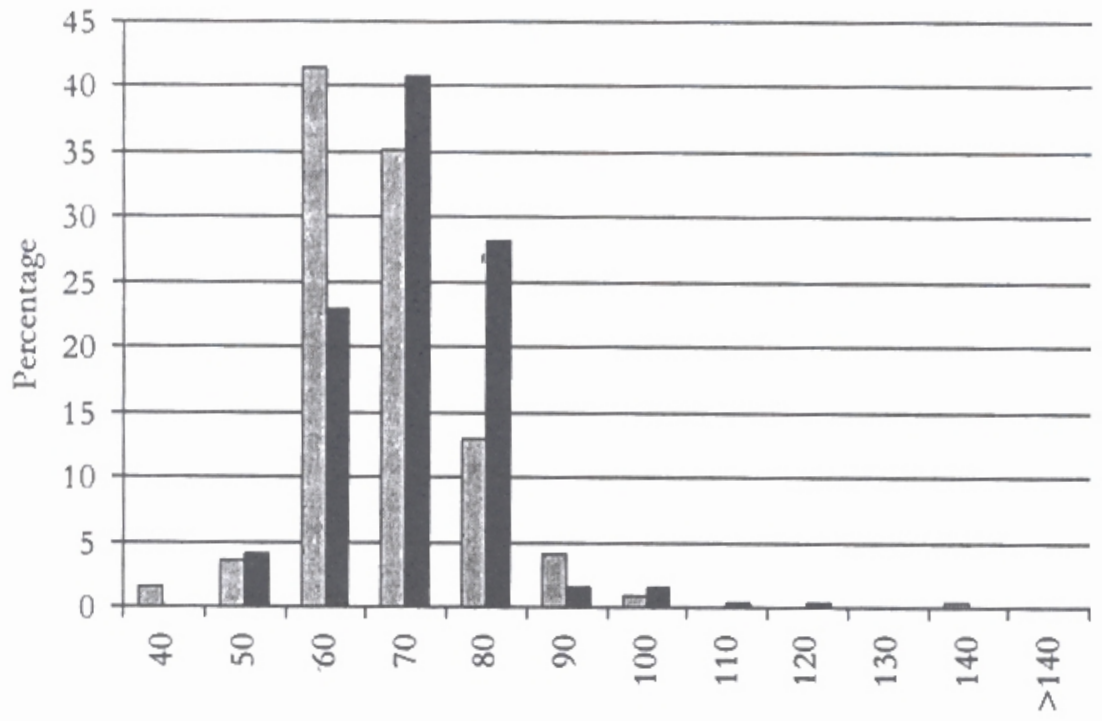

国 Health workers

Observer

Diastolic bood pressure

Figure 16 Distribution of Diastolic Blood Pressure as recorded by Health Workers and Observer in 248 Women 
The agreement between health workers and observers on blood pressures is shown in Table 3. Agreement in individual diagnosis was fair for elevated diastolic, moderate for systolic and moderate for elevated blood pressures as shown by the Kappa coefficients.

About $95 \%$ of the women reported that they were generally satisfied with the care they received. There was no difference in the level of satisfaction between those who received information on the blood pressure results $(94.1 \%)$ and those who did not $(94.9 \%)$.

Table 3 Agreement between Health Workers and Observer Measurements of Elevated Blood Pressure in 248 Women

\begin{tabular}{lcc}
\hline $\begin{array}{l}\text { Elevated blood } \\
\text { pressure } \\
\text { measurement }\end{array}$ & $\begin{array}{c}\text { Kappa } \\
\text { coefficient }\end{array}$ & $\begin{array}{c}\mathbf{9 5 \%} \\
\text { confidence } \\
\text { interval }\end{array}$ \\
\hline Systolic & 0.45 & $0.11-0.79$ \\
Diastolic & 0.32 & $0.06-0.57$ \\
Hypertension & 0.43 & $0.03-0.84$ \\
\hline
\end{tabular}

\section{Discussion}

This cross-sectional study shows that the quality of screening for hypertension in pregnancy was inadequate in the district. The health facilities had inadequate equipment for detecting hypertension, inadequate/inaccurate screening process, and health workers gave poor feedback on the results of investigation to their client.

Early detection of hypertension and protenuria is possible by using relatively simple instruments like stethoscope, sphygmomanometer and a urine albumin test (albustix). These should be a pre-requisite at every level where surveillance of pregnancy hypertension is to be conducted. In our study, although blood pressure and protenuria should be recorded in the local antenatal card (MCH4), the majority $(90 \%)$ of peripheral dispensaries had no means of performing any urine investigation (albustix). Thus, the major weakness was inadequate structure for screening for hypertension in pregnancy.
The most important task of antenatal care is to achieve adequate risk assessment. In our study, screening for hypertension in pregnant women was not done in 35\% of the women attending ANC facilities in the district. A similar study on quality of risk factor screening in Niger $^{13}$ reported that $44 \%$ of women attending antenatal care were not examined for blood pressure by midwives despite availability of necessary equipment in all institutions that participated in the study. Other reports have shown that only a low proportion of risk factors are detected at antenatal clinics. ${ }^{14}$ A survey of eclamptic women in United Kingdom in 1992 demonstrated that more than one third of women developed seizures in hospital, with neither hypertension nor protenuria being documented even though they may have been present. ${ }^{15}$

A considerable disagreement was found between measurements made by health workers and observers. A similar result was reported in Niger, where disagreement between the reference examiner and midwives was high for many risk factors. ${ }^{13}$ Systematic errors in routine recording of blood pressure measurement have been reported, ${ }^{16}$ indicating failure in standardising method and/or terminal digit preference. Other errors could be due to the use of other instrument rather than manual mercury sphygmomanometer or the use of different Korotkoff sounds. We could not assess whether all health workers recorded blood pressure at the same Korotkoff sounds as our observer.

Protenuria is widely acknowledged as a reliable prognostic risk factor for adverse maternal and perinatal outcomes; ${ }^{17}$ thus, the accuracy of measuring protenuria is a crucial point in the management of hypertensive disease in pregnancy (HDP). If dipsticks are used for screening, it is advised that protenuria in HDP should always be confirmed in a 24-hour quantitative protein of urine collection. ${ }^{16}$ In our study this investigation was only possible in RHCs and hospitals.

Improved detection and care for women with hypertensive disease in pregnancy (HDP) have improved maternal outcomes. There is, however, little evidence to suggest which specific interventions would be effective. Most eclamptic fits in UK still occur in hospitals, indicating that early detection and 
treatment are not effective in preventing disease progression. ${ }^{15}$ In Sweden, analysis of historical data suggested that early fall in mortality from eclampsia primarily due to improved case survival might be a result of better treatment of advanced disease. ${ }^{18}$ The above findings can indicate low reliability of antenatal care in respect to prediction and management of obstetric problems, and this has been demonstrated in both developed and developing countries. ${ }^{14}$

There's need for further research on factors that determine health workers' motivation. The study conducted in Niger ${ }^{13}$ found that midwives claimed lack of time as reason for not searching for risk factors that could be detected by physical and/or biological examination including blood pressure measurements. The fact that two thirds of pregnant women had their blood pressure checked but only 9\% were informed about their result in our study shows poor communication between provider and client even in hospitals and RHCs. A study on anaemia in pregnancy in similar settings also reported poor communication, but peripheral clinics were more likely to give information to the women than the large overcrowded clinics at the hospital. ${ }^{19}$ Studies on drug compliance emphasise that regardless of which type of practitioner is consulted, communication between provider and client is very important and has considerable effect on client compliance. ${ }^{20}$ Since only $8.0 \%$ of women with hypertension received the expected quality of care, very little impact can be expected on maternal morbidity or mortality due to screening for hypertension in pregnancy.

Responses from the women on the level of satisfaction with care do not seem to correspond to the actual experience or professional expectation from the programme. This indicates presence of poor knowledge among women about hypertension in pregnancy and what to expect from the antenatal care services. It has been suggested that increased public awareness of the danger signs and need for referral for pre-eclampsia/eclampsia may contribute to a reduction in mortality due to this condition. ${ }^{2} \mathrm{~A}$ qualitative method would be more appropriate to understand women's perspectives on their satisfaction.
Considering the poor performance of ANC in detection and management of HDP, preventive activities might be more appropriate especially in lowincome settings. Systematic reviews of interventions to prevent pre-eclampsia and its sequelae indicate that calcium supplementation during pregnancy might reduce the prevalence of hypertension and pre-eclampsia in women at high risk of gestational hypertension in communities with low dietary calcium intake. ${ }^{16}$

The treatment of established HDP is not part of routine antenatal care given by primary care health workers. Pregnant women must be referred to the first level at which they can receive specialist professional care. Debate on the treatment of HDP still centres on the role of bed rest, antihypertensives and anti-convulsants. While there is little evidence of effectiveness of bed rest for treating pre-eclampsia, anti-hypertensive drugs in women with systolic blood pressure $>169 \mathrm{mmHg}$ and/or diastolic blood pressure $>109 \mathrm{mmHg}$ is reported to be beneficial. ${ }^{16}$ The appropriateness of prophylactic anti-convulsants in treating preeclampsia is not clear, but magnesium sulphate is reported to be more effective than diazepam, phenytoin or lytic cocktail in the treatment of eclampsia. ${ }^{16}$ In our study, no evaluation was done at the hospital level to assess the different regimens used for treatment of pre-eclampsia, but at least the hospitals had some drugs available for initial treatment.

Our study has highlighted practical limitations in providing good quality screening of hypertension in pregnancy in a rural developing country. Inadequate facilities for screening, poor reliability of blood pressure measurements during screening, low motivations of health providers are important problems to be addressed. The lesson from historical data in Sweden where a fall in mortality from eclampsia was due to improved case survival as a result of better treatment of advanced disease suggests that the ultimate impact of antenatal efforts will be seen when essential obstetric care of good quality is put in place in all facilities so as to manage complications due to hypertension in pregnancy. 


\section{Acknowledgement}

We thank Prof. Siriel Massawe of the Department of Obstetrics and Gynaecology, MUCHS, for her field guidance. We are grateful to our research assistants, district medical officer, health workers and women who supported and participated in the study. This study was funded by the Swedish Agency for Research and Co-operation with Developing Countries (SAREC) under collaboration between MUCHS, Tanzania, and the Uppsala and Umea Universities of Sweden.

\section{REFERENCES}

1. Oney T and Meyer-Sebellek W. Variability of arterial blood pressure in normal and hypertensive pregnancy. J Hypertension 1990; 8(suppl 6): 577-581.

2. World Health Organization. Antenatal Care. Report of a Technical Working Group. Geneva: WHO, 1996 a.

3. Halligan A, O’Brien E, O’Malley K, Darling M and Walshe J. Clinical application of ambulatory blood pressure in pregnancy. J Hypertension 1991; 9(Suppl 8): $575-577$.

4. Bureau of Statistics M-II. Tanzania Demographic Health Survey 1996. Calverton: Bureau of Statistics and Macro International, 1996.

5. Urassa EJN, Lindmark $G$ and Nystrom L. Maternal mortality in Dar es Salaam, Tanzania: Socioeconomic, obstetric history and accessibility of health care factors. Afr J Health Sci 1995; 2: 242249.

6. World Health Organization. New estimates for maternal mortality. Wkly Epidemiol Rec 1996; 71: $97-$ 104.

7. Macleod J and Rhode R. Retrospective follow-up of maternal deaths and their associated risk factors in a rural district of Tanzania. Trop Med Int Health 1998; 3: 130-137.
8. Urassa EJN. Pregnancy outcome in patients presenting with hypertension in pregnancy in Muhimbili Medical Centre. J Obst Gyn East Cent Afr 1984; 3: 55-57.

9. Urassa EJN, Massawe S, Lindmark G and Nystrom L. Maternal mortality in Tanzania - medical causes are interrelated with socio-economic and cultural factors. S Afr Med J 1996; 86: 436-444.

10. UNICEF, WHO, UNFPA. Guidelines for Monitoring the Availability and Use of Obstetric Services. 2nd Edition. New York: UNICEF, 1997.

11. Kielmann AA, Janovsky $\mathrm{K}$ and Annett H. Assessing District Health Needs, Services and Systems: Protocols for Rapid Data Collection and Analysis. London: Macmillan and AMREF, 1995.

12. Altman GD. Practical Statistics for Medical Research. London: Chapman and Hall, 1991: 396-409.

13. Prual A, Toure A, Huguet D and Laurent Y. The quality of risk factor screening during antenatal consultations in Niger. Health Policy Plann 2000; 15: 11-16.

14. Rooney C. Antenatal Care and Maternal Health: How effective is it? Geneva: WHO, 1992.

15. Douglas K and Redman C. Eclampsia in the United Kingdom. Br Med J 1994; 309: 1264-67.

16. Carroli G, Rooney $\mathrm{C}$ and Villar J. WHO programme to map the best reproductive health practices: How effective is antenatal care in preventing maternal mortality and serious morbidity? Paed Perinat Epidemiol 2001; 15(Supplement 1): 1-42.

17. Halligan AWF, Bell SC and Taylor DJ. Dipstick protenuria: caveat emptor. Br J Obstet Gynaecol 1999; 109: 1177-80.

18. Hogberg $U$ and Joelsson I. The decline in maternal mortality in Sweden, 1931-1980. Acta Obstet Gynecol Scand 1985; 64: 583-592.

19. Massawe S, Urassa E, Lindmark G and Nystrom L. Anaemia in pregnancy: perceptions of patients in Dar es Salaam. East Afr Med J 1995; 72: 498-503.

20. Nyanzema NZ. Towards better patient drug compliance and comprehension: a challenge to medical and pharmaceutical services in Zimbabwe. Soc Sci Med 1984; 18: 551-554. 